\title{
La contribución de los fósiles a la reconstrucción de las dinámicas de población de murciélagos
}

\author{
Juan Manuel LóPez-García ${ }^{1,2}$ Y Paloma SeVIlla ${ }^{3}$
}

${ }^{1}$ IPHES, Institut Català de Paleoecologia Humana i Evolució Social,

C/Escorxador s/n, E- 43003 Tarragona, Spain

${ }^{2}$ Àrea de Prehistòria, Universitat Rovira i Virgili (URV)

Avinguda de Catalunya 35, E-43002 Tarragona, Spain.

${ }^{3}$ Departamento de Paleontología, Facultad de Ciencias Geológicas (UCM) e Instituto de Geología Económica (CSIC)

C/ Jose Antonio Novais 2, E-28040 Madrid, Spain. psevilla@geo.ucm.es.

Correo electrónico del autor: jmlopez@iphes.cat

DOI: http://dx.doi.org/10.14709/BarbJ.5.1.2012.02

English title: he role of fossils in the reconstruction of bat population dynamics

\begin{abstract}
Modern research in the conservation biology of bats enables us to establish the status of these mammals on the basis of their demography and population dynamics. Data obtained from bat remains found in fossil and sub-fossil localities provide the means for this long-term tracking of populations, of which many good examples are available in published papers. In this paper we present an overview of how bat fossils can be useful in conservation biology and give a number of examples. As well, we discuss the limitations of the use of these mammals for this purpose.
\end{abstract}

Keywords: fossil Chiroptera, Quaternary population dynamics, past human-bat interactions

Resumen: Las investigaciones recientes en la conservación de murciélagos permiten establecer el estatus de estos mamíferos en base a su demografía y la dinámica de poblaciones. Los datos obtenidos de los restos de murciélagos encontrados en localidades fósiles y subfósiles proporcionan los medios para el seguimiento de estas poblaciones a largo plazo, muchos buenos ejemplos se encuentran disponibles en artículos publicados. En este trabajo, vamos a presentar una visión general de cómo los fósiles pueden ser útiles en la biología de la conservación de murciélagos con algunos ejemplos, así como los límites de la utilización de estos mamíferos para el propósito discutido.

Palabras clave: Quirópteros fósiles, dinámicas de población en el Cuaternario, interacción humanos-murciélagos en el pasado

\section{INTRODUCCIÓN}

En el presente artículo se explica el procedimiento habitual en el estudio de los murciélagos fósiles con el fin de obtener la información ecológica del pasado. Además explicamos brevemente las modificaciones que se producen en los conjuntos fósiles (tafocenosis) que difiere de la biocenosis original, seguido por los métodos actuales que se utilizan para la identificación de los murciélagos fósiles y la información ecológica que puede inferirse de estos, con ejemplos seleccionados de trabajos publicados. Por último se muestran algunos ejemplos de las tendencias particulares que pueden inferirse a partir del registro del Cuaternario sobre especies europeas actuales de murciélagos.

\section{DE LAS COMUNIDADES VIVAS A LAS ASOCIACIONES FÓSILES}

Los murciélagos se incluyen dentro de un grupo llamado de forma informal "micromamíferos", que como característica general no superan los $5 \mathrm{~kg}$ de peso (Merritt 2010). La separación entre grandes mamíferos y micromamíferos es muy útil en paleontología, ya que la fosilización de los mamíferos varia generalmente en función a su tamaño. Existen dos tipos de localidades donde se pueden encontrar fósiles de murciélagos, las formaciones lacustres o ambientes fluviales y las cavidades subterráneas (Fig. 1).

Los restos de murciélagos son menos comunes en el primer tipo de yacimientos, mientras que los rellenos en fisuras y 
en depósitos de cuevas en ambientes kársticos son los sitios más favorables para encontrar fósiles de murciélagos (Sigé \& Legendre 1983).

Por otro lado, los murciélagos son frecuentes en las cuevas y las fisuras de las rocas que utilizan como refugios, en éstas, muchas especies hibernan y se reproducen, formando a veces colonias de grandes grupos. La muerte natural se suele producir en muchas ocasiones mientras los murciélagos hibernan. Después de que el cadáver haya sufrido deterioro y esté cubierto por sedimento, el esqueleto tiene una alta probabilidad de preservación y fosilización. Como resultado, las fisura y cuevas kársticas son los lugares más propicios para encontrar murciélagos fósiles en abundancia.

\section{PReSERVACIÓN DE LOS MURCIÉLAgos FósILES}

Son bien conocidos los ejemplos de esqueletos de murciélagos fósiles completos o prácticamente completos, como los de Messel en Alemania (Habersetzer y Storch 1987) o la Green River Formation en Wyoming (Jepsen 1966). Las ventajas de esta excepcional preservación no es que sólo todas las partes anatómicas del esqueleto estén representadas, sino que nos proporcionan datos precisos para establecer relaciones taxonómicas, evolutivas e información ecológica que de otra forma serían difíciles de obtener. Información acerca de las características tales como, el tipo de vuelo o el desarrollo de la ecolocalización se obtienen a partir de estos fósiles (Habersetzer et al 1994, Simmons y Gaisler 1998, Simmons et al. 2008).

Sin embargo, como sucede con otros vertebrados, la desarticulación de la estructura ósea se produce durante el proceso de fosilización. De esta forma, los huesos aislados y dientes son los elementos más comunes que encontramos en las asociaciones de murciélagos fósiles. La acción de insectos y animales carroñeros, el pisoteo de los animales en la cueva o la acción del agua que circula en el sistema kárstico contribuyen a la dispersión de los huesos y pueden conducir a la perdida de los huesos más pequeños y delicados. En consecuencia, las mandíbulas, maxilares, dientes y húmeros son los restos más frecuentes y mejor representados en los conjuntos de murciélagos fósiles (Fig. 2). Otras partes del esqueleto, tales como omóplatos, pelvis, fémur, la cóclea o fragmentos de falanges se encuentran también, en menor media, representados en estas agrupaciones. Los métodos adecuados de recolección que evitan una representación sesgada hacia las especies más comunes o de mayor tamaño son esenciales para prevenir la pérdida de información.

\section{DETERMINACIÓN TAXONÓMICA DE LOS MURCIÉLAGOS FÓSILES}

Los huesos de murciélagos son fáciles de reconocer en un conjunto fósil de otros huesos de pequeños mamíferos, la adaptación al vuelo es evidente en los huesos largos y delgados; los dientes presentan el patrón típico esperado para su dieta insectívora (en la mayoría de los murciélagos). Sin embargo, la identificación a nivel de género o especie no siempre es posible y, depende de los elementos anatómicos esqueléticos disponibles en el conjunto.

Las alas se encuentran entre las partes más especializadas de los murciélagos, los huesos de estás son alargados y ligeros, distinguibles fácilmente de otros huesos de mamíferos. Sin embargo, a excepción del húmero, no se pueden relacionar con ninguna especie en concreto. Por otra parte, el húmero (Fig. 3) de los murciélagos ha desarrollado en la articulación del codo un dispositivo de bloqueo especializado que difiere entre los diferentes grupos de murciélagos (Felten et al 1973, Hand et al 2009).

Los restos craneales y mandibulares son relativamente resistentes a los procesos destructivos durante la fosilización. Si una fila dentaria se conserva completa, la fórmula dental nos puede ayudar a reconocer los géneros. La forma de los dientes, la diferencia de tamaño entre los premolares anteriores, el grado de compresión de la hilera de dientes, así como el tamaño general, permite la identificación a nivel de especie (Fig. 3). Los dientes aislados son los fósiles más comunes de encontrar en una asociación. En estos casos la determinación de las especies se basa principalmente en las piezas dentarias más características (sobre todo los dos primeros molares superiores y en menor medida los molares inferiores, premolares y caninos). Existen diversas publicaciones sobre la determinación de las especies de murciélagos fósiles europeas en base a su morfología dentaria (Menú y Sige 1971, Sevilla 1986, Menú y Popelard 1987). Además, la comparación directa con material actual es útil, ya que algunas especies han experimentado cambios de tamaño durante el Pleistoceno.

\section{TIPOS DE ASOCIACIONES FÓSILES}

Los fósiles de murciélagos encontrados en localidades kársticas generalmente, aunque no siempre, pertenecen a animales muertos en el interior de la cavidad ("tanatocenosis"). Una asociación en cueva de murciélagos se caracteriza generalmente por una o dos especies dominantes y número variable de especies representadas en menor medida que las acompañan. Las colonias de cría de murciélago pueden ser identificadas cuando se encuentran dientes deciduos, una cantidad considerable de huesos sin osificar o dientes sin desgaste muy abundante. Por otro lado, el guano generado por las colonias de estivación de murciélagos al ser muy ácido puede llegar a destituir los huesos (Kowalski 1995). En este caso la presencia de este tipo de colonias se puede detectar mediante las características de los sedimentos, alterados por el guano. Además, las colonias de hibernación pueden ser reconocidas por la menor proporción de individuos con grados intermedios de desgaste dental, en comparación a los individuos con una desgaste dental acusado (individuos seniles) o poco desgastado (individuos juveniles).

Es bastante frecuente hallar conjuntos en los que se encuentran restos de murciélagos junto con los de otros vertebrados pequeños que no viven en cuevas. Esta asociación se interpreta comúnmente como origen de la acumulación de los restos de la dieta de un predador, evidenciados por los patrones de fractura y digestión (Andrews 1990). Por lo general, los murciélagos rara vez se encuentran en egagrópilas o defecaciones de predadores, pero hay casos en que se encuentran pruebas de digestión en los huesos de murciélagos, como es el caso de Matuzka Cave (Rossina et al 2006). Estos murciélagos procedentes de conjuntos de presas proporcionan información útil a cerca de especies poco comunes en las cuevas y contribuyen al conocimiento de estas especies en el pasado, como Tadarida, Eptesicus o Nyctalus. 


\section{DENSIDAD Y DIVERSIDAD INFERIDAS CON MURCIÉLAGOS FÓSILES}

Debido al proceso de fosilización, el registro fósil da pocas oportunidades para inferir directamente cuantos animales se encontraban en una comunidad viva de los que murieron y se convirtieron en parte del conjunto fósil. En el caso de los murciélagos que se encuentran en cuevas, la posibilidad de un sesgo hacia una especie en particular es poco probable, un número elevado de una especie en particular puede ser indicativo de la presencia de numerosos individuos de esa especie. Teniendo en cuenta que los esqueletos fosilizados de los vertebrados se suelen conservar desarticulados, un individuo puede estar representado en una asociación por diferentes elementos fósiles. El uso de índices, como el número mínimo de individuos (NMI) permite contrarrestar el sesgo que se obtiene si la abundancia se calcula directamente del número de restos (NR) identificados (Lyman 1987, Marshall y Pilgram 1993). A pesar de que el NMI no le da un valor absoluto a la abundancia, como ocurre cuando se trabaja con animales vivos (Plug y Plug 1990), es una manera útil para comparar la representación de especies en diferentes momentos dentro de la misma localidad, entre las diferentes localidades fósiles, o para establecer cambios en la abundancia a través del tiempo. Por otra parte, ya que las asociaciones fósiles de murciélagos rara vez son constituidas por una sola especie, el NMI ofrece la posibilidad de establecer valores fiables de las abundancias relativas de las diferentes especies que constituyen una comunidad.

\section{Cambios en las abundancias a lo largo de UNA SECUENCIA}

La sedimentación en cuevas o en fisuras kársticas puede durar miles de años, durante los cuales se suceden cambios climáticos significativos. Los factores de temperatura y humedad determinan las diferencias entre los sedimentos de las cuevas, así como su contenido fósil. Los cambios climáticos que en el pasado han provocado modificaciones en la vegetación local han influido en la distribución y abundancia de murciélagos. Por ejemplo, durante las fases climáticas frías del Pleistoceno superior buena parte de España estaba cubierta de vegetación de estepa fría (Sánchez Goñi et al. 2002), con la mejora del clima a comienzos del Holoceno, las áreas forestadas se hicieron más abundantes y una mayor variedad de tipos de vegetación se extendió en la península (Riera Mora 2006). En consecuencia, en determinadas secuencias sedimentarias de cuevas, como El Mirador (Burgos, España) y la Cova Colomera (Cataluña, España), que abarcan una cronología desde el Pleistoceno superior final a principios del Holoceno, se observa un aumento en la abundancia y diversidad de murciélagos. Mientras que en los niveles del Pleistoceno superior aparecen representadas sólo unas pocas especies muy tolerantes, como Myotis myotis, Miniopterus schreibersii y Pipistrellus pipistrellus, los niveles del Holoceno se enriquecen con especies termófilas y forestales, tales como Rhinolophus euryale, R. mehelyi y las especies del género Plecotus (LópezGarcía 2008, López-García et al 2008, Oms et al 2009). Ejemplos similares se conocen en otros lugares de Europa, donde las especies más pequeñas del género Myotis dominan las asociaciones durante el Cuaternario. Por ejemplo, la secuencia del Pleistoceno superior- holoceno de Komarowa en Polonia (Ochman 2003) se caracteriza por un cambio de la dominación de M. daubentonii en las capas del Pleistoceno y $M$. bechsteinii en las capas del Holoceno, acompañado de aumento en la abundancia y la diversidad de murciélagos. Del mismo modo, Postawa (2004), después de estudiar los fósiles de murciélagos de 11 cuevas con sedimentos del Holoceno en el sur de Polonia, concluyó que los cambios en la composición de especies mostraban una alta correlación con las fluctuaciones climáticas y los cambios de vegetación que tuvieron lugar durante las fase del Atlántico y del Subboreal.

\section{CAMbios en la distribuCión geOgRÁFICA}

Los depósitos en cuevas y los rellenos de fisuras no siempre contienen una secuencia que representa diferentes episodios sedimentarios bajo diferentes condiciones ambientales. Un evento único de relleno puede suceder, sin dar más información acerca de las condiciones anteriores o posteriores. La información proporcionada por un conjunto único sigue siendo útil para el estudio de la dinámica de la población de murciélagos en el pasado, ya que los fósiles proporcionan pruebas de la presencia de estas especies en la zona. En ocasiones, los registros de las especies fuera de su distribución actual proporcionan información respecto a su distribución en el pasado, o los cambios causados por cambios climáticos. Entre los ejemplos disponibles de estos cambios en la distribución tenemos el de Myotis dasycneme, actualmente distribuido en el norte de Europa, que se encuentra presente en localidades del Pleistoceno medio y superior de Francia (Mein 1975, Clot y Duranthon 1990) o en el norte y centro de Italia (Tata y Kotsakis 2005; Argenti et al. 2008). Otros ejemplos se dan en los registros de Eptesicus nilssonii en el Pleistoceno medio y final de Francia (Mein 1975, Noel et al. 2008) (Fig. 4).

\section{INFLUENCIA HUMANA EN LAS ABUNDANCIAS Y DIVERSIDAD DE MURCIÉLAGOS}

Las cuevas utilizadas como refugios para murciélagos han sido compartidas con los seres humanos en el pasado. Existen localidades del Pleistoceno medio y superior donde se produce una disminución en la abundancia de murciélagos en los niveles donde hay evidencias de ocupación humana intensiva. En ocasiones, se puede inferir el uso estacional de las cuevas, los seres humanos ocupan las cuevas durante el invierno, dejando como evidencia hogares, huesos de grandes mamíferos con marcas que indican la carnicería, así como industria lítica, mientras que los murciélagos habitan en las cuevas durante el verano, una vez libre de la ocupación humana. Un ejemplo de esto se describe en las secuencia de la cueva Denisova en las montañas de Altai (Rossina 2006). Los niveles del Pleistoceno anteriores a la ocupación humana se caracterizan por una importante representación de fósiles de murciélagos con ocho especies diferentes, mientras que en los niveles de ocupación humana de la cueva durante el Pleistoceno superior el número de murciélagos disminuye a excepción de Myotis blythii. Por otro lado, los niveles de la cueva de Matuzka (Rossina et al. 2006), indican las visitas regulares y a corto plazo de los humanos durante el Pleistoceno Superior, estas ocupaciones a corto plazo se intercalan con las ocupaciones de las comunidades de murciélagos. Otro ejemplo de la interacción entre los humanos y los murciélagos en el Pleistoceno lo encontramos en los niveles del Pleistoceno superior de la cueva de Arene 
Candide (Salari 2010), donde la abundancia de murciélagos se encuentra inversamente correlacionada con los restos encontrados de grandes mamíferos (introducidos en la cavidad por los seres humanos).

Con la mejora climática a partir del Holoceno y el abandono de las cuevas por parte de los seres humanos, las secuencias de las localidades Holocenas muestran un mayor número de restos de murciélagos. A lo largo de tiempos históricos, muchas especies de murciélagos se han beneficiado de la modificación antrópica del paisaje. Las construcciones humanas han proporcionado refugios nuevos, permitiendo a las especies colonizar refugios no naturales. El desarrollo de la agricultura favoreció la expansión de las especie de forraje sobre la vegetación baja, pero al mismo tiempo, la tala de áreas forestales para la agricultura ha tenido un impacto negativo sobre la abundancia de las especies forestales de murciélagos. La evidencia de estos cambios se registra, por ejemplo, en los niveles sedimentarios de la Grotte Rochefort en el noroeste de Francia (Noel et al. 2008), donde los niveles inferiores datan del Pleistoceno y los superiores del siglo XIX.

\section{CONCLUSIONES}

Dado que la mayoría de las especies de murciélagos de la fauna europea moderna ya se encontraban establecidos en Europa desde hace aproximadamente $2 \mathrm{Ma}$, los fósiles conservados en las localidades del Pleistoceno y Holoceno pueden proporcionarnos una visión a largo plazo de las variaciones sufridas por las poblaciones de murciélagos, la abundancia y la distribución de las mismas. Para este propósito es necesario un registro fósil que cubra diferentes áreas geográficas, con el fin de establecer las variaciones geográficas, y que abarque periodos cronológicos distintos con condiciones ambientales diferentes para las consecuencias de estas variaciones en las poblaciones de murciélagos. Los datos disponibles de los murciélagos durante el Cuaternario ha dado lugar a logros como el reconocimiento de las tendencias a largo plazo de determinadas especies. La evidencia fósil muestra que algunas especies comunes en localidades del Pleistoceno y Holoceno, como Myotis nattererii o Myotis myotis, eran comunes en el pasado, y siguen siendo comunes en el presente, dando pruebas de la estabilidad a largo plazo de sus poblaciones. Otras especies como Myotis bechsteinii o Miniopterus schreibersii también son comunes en las asociaciones del Pleistoceno, pero se encuentran bajo una tendencia decreciente desde el Holoceno. Algunas especies poco comunes durante el Pleistoceno como Nyctalus, Tadarida teniotis o Myotis capaccinii siguen siendo hoy día especies raras, lo que indica que, aunque son poco frecuentes, sus poblaciones son bastante estables en una escala paleontológica. Finalmente, los ejemplos de un número creciente de especies de murciélagos se encuentran con los taxones Pipistrellus pipistrellus o Eptesicus serotinus, raros en las localidades durante el Pleistoceno, pero en la actualidad bastante abundantes, favorecidos por las actividades humanas.

\section{Agradecimientos}

Este artículo forma parte de los proyectos CGL2009-07896/ BTE and SGR2009-324. J.M.L.-G ha sido apoyado por una beca postdoctoral del Subprograma Juan de la Cierva (JCI2009-04026), con el financiamiento del Ministerio de Ciencia e Innovación. P.S. es miembro del grupo de investigación GR58/08-910607.

\section{REFERENCIAS}

Andrews, P. 1990. Owls, caves and fossils. Predation, preservation and accumulation of small mammal bones in caves, with an analysis of the Pleistocene Cave faunas from Westbury-sub-Mendip, Somerset, UK. Natural History Museum. London. 239 pp.

Argenti, P., Kotsakis, T., SAbatini, F. 2008. Myotis dasycneme in a latest Pleistocene assemblage of Cittareale Cave (Rieti, Latium, Central Italy). Geologica Romana, 41, 117-123.

Clot, A., Duranthon, F. 1990. Les Mammifères fossiles du Quaternaire dans les Pyrénées. Museum d'Histoire Naturelle de Tolouse. 159 pp. DOI: https://dx.doi. org/10.1016/0016-6995(91)80045-2

Felten, H., Helfricht,A., Storch, G. 1973. Die Bestimmung der Europäischen Fledermausfaunen nach der distal epyphise des Humerus. Senckenbergiana biologae 54, 291-297.

Habersetzer, J., Storch, G. 1987. Klassifikation und funktionelle Flügelmorphologie paläogener Fledermäuse (Mammalia, Chiroptera). Courier Forschungsinstitut Senckenberg, 91, 117-150.

Habersetzer, J., Richter, G., Storch, G. 1994.Paleoecology of early middle Eocene bats from Messel, FRG. aspects of flight, feeding and echolocation. Historical Biology, 8, 235-260. DOI: https://dx.doi. org/10.1080/10292389409380479

Hand, S.J., Weisbecker, V., Beck, R.M.D., Archer, M., Godthelp, H., Tennyson, A.J.D., Worthy, T.H. 2009. Bats that walk: a new evolutionary hypothesis for the terrestrial behaviour of New Zealand's endemic mystacinids. BMC Evolutionary Biology, 9,1471-2148. DOI: https://dx.doi.org/10.1186/14712148-9-169

JEPSEN, G.L. 1966. Early Eocene Bat from Wyoming. Science, 154, 1333-1339. DOI: https://dx.doi.org/10.1126/ science. 154.3754 .1333

KowALSKI, K. 1995. Taphonomy of bats (Chiroptera). Geobios, M.S. 18, 251-256. DOI: https://dx.doi. org/10.1016/S0016-6995(95)80172-3

LóPEZ-GARCíA, J.M. 2008. Evolución de la diversidad taxonómica de los micromamíferos en la Península Ibérica y cambios Paleoambientales durante el Pleistoceno superior. Tesis Doctoral. Universitat Rovira i Virgili, Tarragona. 368 pp. 
López-García J.M. Cuenca-Bescós G, Rosell J. 2008 Resultados del estudio de microvertebrados del Neolítico de la Cueva de El Mirador (Ibeas de Juarros, Sierra de Atapuerca, Burgos). In: Hernández MS, Solé JA, López JA (eds) Actas IV Congreso de Neolítico Peninsular. Museo Arqueológico de Alicante, Alicante, pp 338-344.

LYMAN, R. L. 1987. On the analysis of vertebrate mortality profiles: sample size, mortality type and hunting pressure. American Antiquity, 52, 125-142

Marshall, F., PILgram, T. 1993. NISP vs. MNI in quantification of body-pPart representation. American Antiquity, 58, 261-269.

MeIN, P. 1975. Les Chiroptères (Mammalia) du gisement Pléistocène moyen des Abimes de la Fage à Noailles (Corrèze). Nouvelle Archives du Musée d'Histoire naturelle de Lyon, 13, 57-67.

Menú H., Popelard, J.B., 1987. Utilisation des charactères dentaires pour la détermination des vespertilioninés de l'ouest européen. Le Rhinolophe 4, 1-88.

Menú H., Sigé, B., 1971. Nyctalodontie et myotodontie, importants caractères de grades évolutifs chez les Chiroptères entomophages. Comptes Rendus de l'Academie des Sciences de Paris, 272, 1735-1738.

Merritt, J.F. 2010. The Biology of Small mammals. John Hopkins University Press. Baltimore. 368 pp.

Noel, F., Chaut, J.-J. \& Hinguant, S. 2008. 11000 ans d'histoire des chiroptères dans la Grotte Rochefort (Mayenne, France). In: Société française d'études et de protection des mammifères. Symbioses (n.s.) 21, 13-15.

Ochman, K. 2003. Late Pleistocene and Holocene bats (Chiroptera) from the Komarowa Cave (CracowCzestochowa Upland, Poland)- preliminary results. Acta Zoologica cracoviensia, 46, 73-84.

Oms FX, Petit MA, Allué E, Bargalló A, Blain H-A, López-García JM, Martin P, Morales JI, Pedro M, Rodriguez A, Solé A. 2009 Estudio trasndisciplinar de la fosa EE1 de la Cova Colomera (Prepirineo de Lleida): implicaciones domésticas y paleoambientales en el Bronce Antiguo del Noreste de la Península Ibérica. Trabajos de Prehistoria, 66, 123-144. DOI: https://dx.doi.org/10.3989/ tp.2009.09016

Plug, C., Plug, I. 1990. MNI Counts as Estimates of Species Abundance. The South African Archaeological Bulletin, 45, 53-57.

Postawa, T., 2004. Changes in bat fauna during the Middle and Late Holocene as exemplified by Thanatocoenoses dated with 14C AMS from Kralów-Czestochowa Upland caves, Poland. Acta Chiropterologica, 6(2), 269-292. DOI: https:// dx.doi.org/10.3161/1508110042955487
Riera Mora, S. 2006. Cambios vegetales holocenos en la región mediterránea de la Península Ibérica: ensayo de síntesis. Ecosistemas 15(1):17-30.

Rossina, V.V., 2006. Bats as an Indicator of Human Activity in the Paleolithic, Using the Example of Denisova Cave, Northwestern Altai. Paleontological Journal 40 (4), 494-500. DOI: https://dx.doi.org/10.1134/ $\underline{\mathrm{S} 0031030106100091}$

Rossina, V.V., BAryshnikov, G.F., Woloszyn, B.W., 2006. Dynamics of the Pleistocene bat fauna from the Matuzka Paleolithic site (Northern Caucasus, Russia) (Chiroptera). Lynx, 37, 229-240.

SALARI, L. 2010. Late Glacial bats from the "M" layers of the Arene Candide Cave (Liguria, Italy). Rivista italiana di Paleontologia e Stratigrafia, 116(1), 119138 .

Sánchez Goñi, M.F., Cacho, I., Turon, J.-L., Guiot, J., Sierro, F.J., Peypouquet, J.-P., J.O. Grimalt, Shackelton, N.J. 2002. Synchroneity between marine and terrestrial responses to millennial scale climatic variability during the last glacial period in the Mediterranean region. Climate Dynamics, 19, 95-105. DOI: https://dx.doi.org/10.1007/s00382001-0212-x

SEVILLA, P. 1986. Identificación de los principales quirópteros ibéricos a partir de sus dientes aislados. Valor sistemático de los caracteres morfológicos y métricos dentarios. Doñana Acta Vertebrata. 13, $111-130$

Sigé, B., Legendre, S. 1983. L'Histoire des peuplements de chiroptères du bassin méditerranéen: l'apport comparé des remplissages karstiques et des dépôts fluvio-lacustres. Mémoires de Biospéleologie, 10, 209-225.

Simmons, N.B. GeISLER, J.H. 1998. Phylogenetic Relationships of Icaronycteris, Archaeonycteris, Hassianycteris, and Palaeochiropteryx to Extant Bat Lineages, with Comments on the Evolution of Echolocation and Foraging Strategies in Microchiroptera. Bulletin of the American Museum of Natural History, 235, 1-182. DOI:https://dx.doi.org/10.1038/nature06549

Simmons, N.B., Seymour, K.L., Habersetzaer, J., GunNell, G.F. 2008. Primitive Early Eocene bat from Wyoming and the evolution of flight and echolocation. Nature, $451,818-821$.

Tata, C., Kotsakis, T. 2005. Italian fossil chiropteran assemblages: a preliminary report. Geologia Alpina, 2, 53-60. 


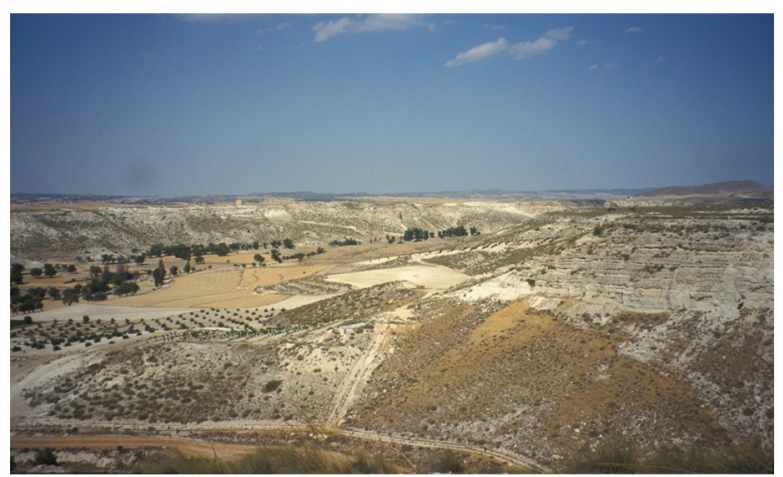

Guadix-Baza Basin-Orce

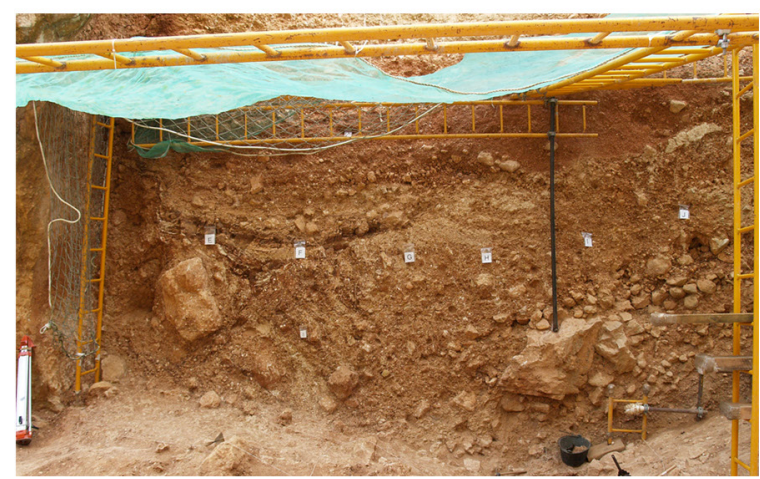

Sima del Elefante-Atapuerca

Fig. 1. Ejemplos de los dos tipos de localidades donde se pueden encontrar murciélagos fósiles. Izquierda: depósitos lacustres de la cuenca de Guadix-Baza (sur de España) (Foto: H.-A. Blain); derecha: Depósito kárstico de la "Sima del Elefante (Atapuerca, Spain) (Foto: J. Rubio).

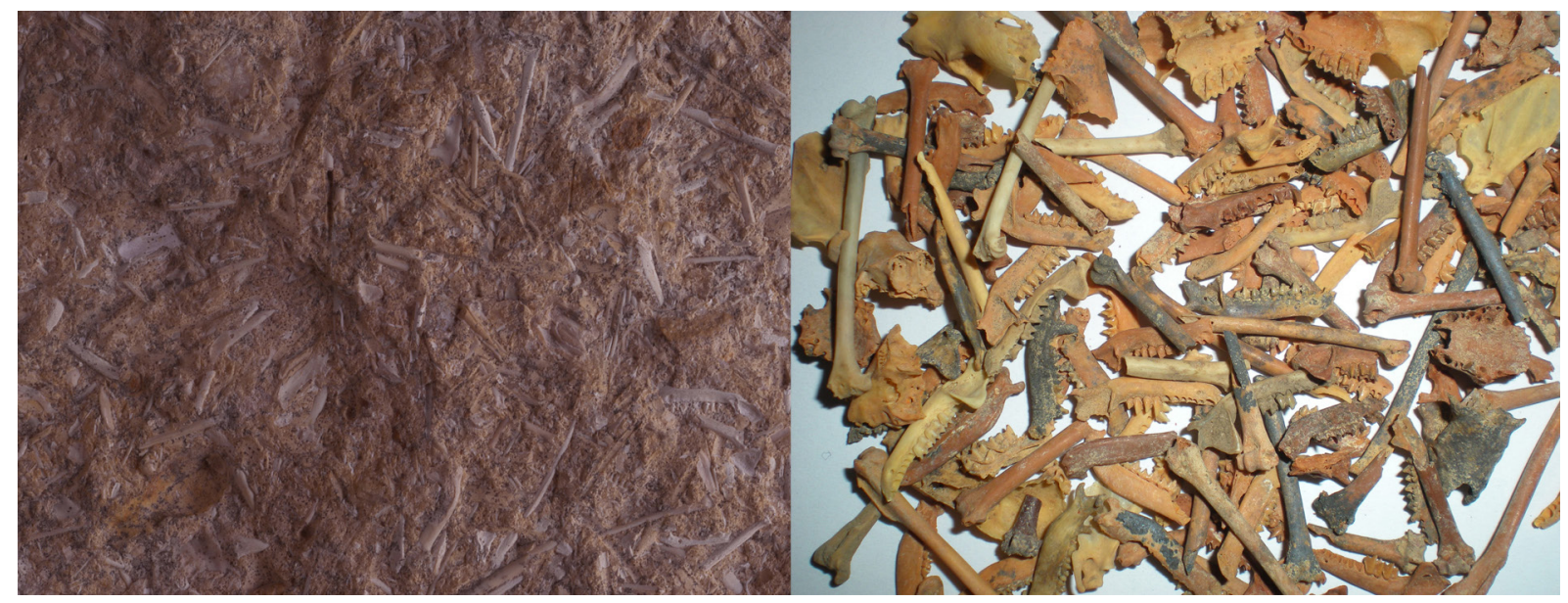

Fig. 2. Izquierda: brecha de huesos procedente de Podlesice (Poland) mostrando la alta densidad de restos de murciélagos. Derecha: Murciélagos fósiles recuperados de los sedimentos en superficie de la Cueva de Azokh (Ngorno Karabagh, Caucaso). Los restos más comunes se pueden observar- mandíbulas, cráneos, escápulas y humeros (Foto: P. Sevilla). 


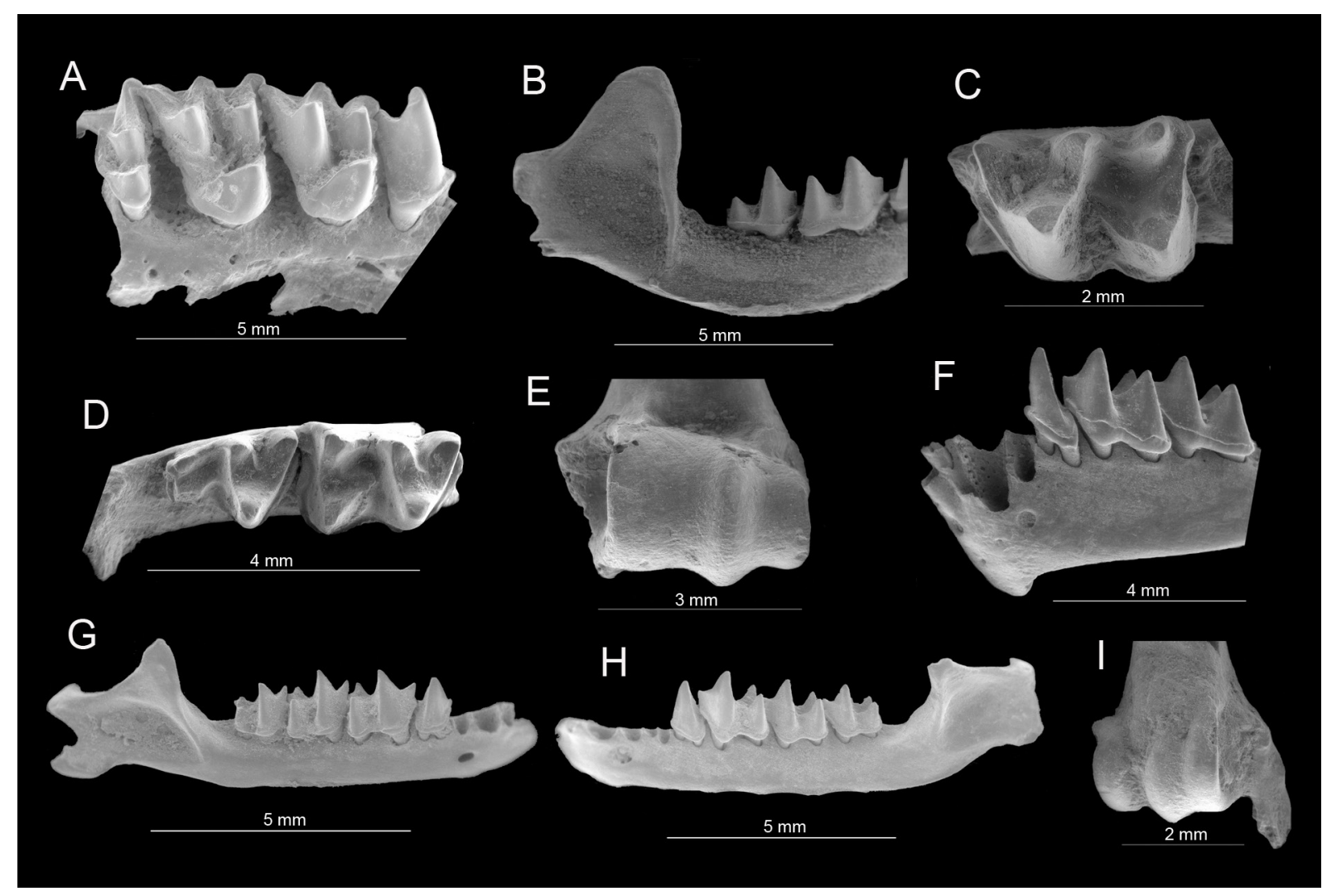

Fig. 3. Algunos restos de murciélagos fósiles. A: maxilar derecho de Myotis myotis (vista oclusal) procedente del Pleistoceno superior de la Cova Colomera (Cataluña, España); B: mandíbula derecha de Myotis myotis (vista labial) procedente del Pleistoceno superior de la Cova del Gegant (Cataluña, España); C: primer molar inferior izquierdo (m1) de Rhinolophus ferrumequinum (vista oclusal) procedente de la Cova del Gegant; D: mandíbula derecha de Myotis myotis (vista oclusal) procedente de Gorham's cave (Gibraltar, UK); E: epífisis distal de húmero de Myotis myotis procedente de la Cova del Gegant; F: mandíbula izquierda de Nyctalus lasiopterus (vista lingual) procedente del Pleistoceno superior del Abric Romaní (Cataluña, España); G: mandíbula derecha de Barbastella barbastellus (vista lingual) procedente de la Cova Colomera; H: mandíbula izquierda de Miniopterus schreibersii (vista lingual) procedente de Gorham's cave; I: epífisis distal de húmero de Miniopterus schreibersii procedente de la Cova del Gegant.
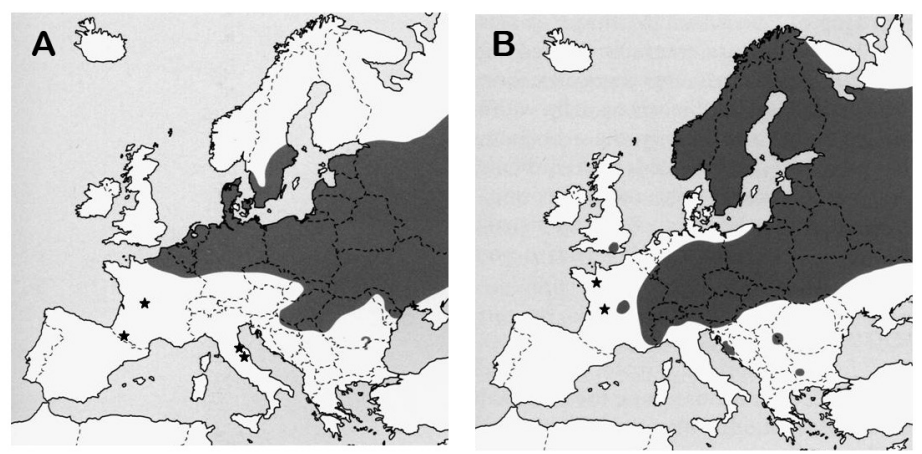

Fig. 4. Ejemplos de cambios en la distribución geográfica de especies de murciélagos durante el Pleistoceno. La distribución actual está marcada en gris, el registro fósil está marcado en asteriscos. A: Myotis dasycneme, B: Eptesicus nilssonii, 